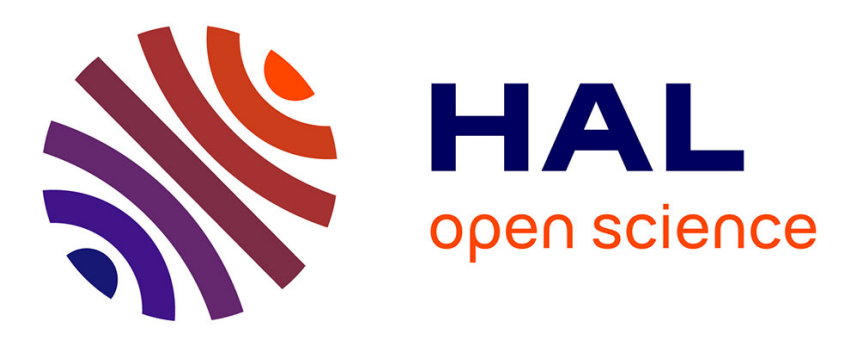

\title{
Linear tetrahedral finite elements for thermal shock problems
}

\author{
Victor D. Fachinotti, Michel Bellet
}

\section{To cite this version:}

Victor D. Fachinotti, Michel Bellet. Linear tetrahedral finite elements for thermal shock problems. International Journal of Numerical Methods for Heat and Fluid Flow, 2006, 16 (5), pp.Pages 590-601. 10.1108/09615530610669120 . hal-00576032

\section{HAL Id: hal-00576032}

https://hal-mines-paristech.archives-ouvertes.fr/hal-00576032

Submitted on 11 Mar 2011

HAL is a multi-disciplinary open access archive for the deposit and dissemination of scientific research documents, whether they are published or not. The documents may come from teaching and research institutions in France or abroad, or from public or private research centers.
L'archive ouverte pluridisciplinaire HAL, est destinée au dépôt et à la diffusion de documents scientifiques de niveau recherche, publiés ou non, émanant des établissements d'enseignement et de recherche français ou étrangers, des laboratoires publics ou privés. 


\title{
LINEAR TETRAHEDRAL FINITE ELEMENTS FOR THERMAL SHOCK PROBLEMS
}

\author{
Víctor D. Fachinotti ${ }^{* \dagger}$ and Michel Bellet ${ }^{*}$ \\ ${ }^{*}$ Ecole des Mines de Paris, Centre de Mise en Forme des Matériaux (CEMEF), \\ UMR CNRS 7635, Sophia Antipolis, France \\ web page: www-cemef.cma.fr \\ ${ }^{\dagger}$ Consejo Nacional de Investigaciones Científicas y Técnicas (CONICET) \\ Centro Internacional de Métodos Computacionales en Ingeniería (CIMEC) \\ Parque Tecnológico del Litoral Centro - Ruta Nacional 168, Paraje El Pozo \\ S3007ABA - Santa Fe, Argentine \\ web page: www.conicet.gov.ar, www.cimec.org.ar
}

\begin{abstract}
Purpose - The paper presents an original method for the numerical treatment of thermal shocks in non-linear heat transfer finite element analysis.

Design/methodology/approach - The three-dimensional finite element thermal analysis using linear standard tetrahedral elements may be affected by spurious local extrema in the regions affected by thermal shocks, in such a severe way to directly discourage the use of these elements.. This is especially true in the case of solidification problems, in which melted alloys at very high temperature contact low diffusive mold materials. The present work proposes a slight modification to the discrete heat equation in order to obtain a system matrix in M-matrix form, which assures an oscillation-free solution.

Findings - The proposed "diffusion-split" method consists basically in using a modified conductivity matrix. It allows for solutions based on linear tetrahedral elements. The performance of the method is evaluated by means of a test case with analytical solution, as well as an industrial application, for which a well-behaved numerical solution is available.

Originality/value - The proposed method should be helpful for computational engineers and software developers in the field of heat transfer analysis. It can be implemented in most existing finite element codes with a minimal effort.
\end{abstract}

Keywords Thermal shocks, heat transfer analysis, finite elements, linear tetrahedra

Paper type Research paper 


\section{INTRODUCTION}

The solution of diffusion problems using the standard finite element method (FEM) is often affected by severe numerical instabilities. These instabilities take for instance the form of unphysical local maxima and minima in solutions expected to be monotonic. Such a spurious behavior evidences a violation of the maximum principle. In fact, when we discretize the diffusion operator using standard (Galerkin) finite elements, the maximum principle is not always satisfied, as discussed by several authors (Putti and Cordes, 1998; Cordes and Putti, 2001; Kosik et al, 2000). The principle satisfied, a maximum/minimum can only occur either initially or at the boundary, in the latter case a flow from/to the outside must exist (Kozic et al, 2000).

The constraints imposed by this principle on numerical modeling are critical in the regions where the solution exhibits steep gradients. In thermal analysis, with the temperature field as solution, we call thermal shocks these steep variations. They usually appear in the boundary vicinity in a domain initially hot (or cold) that is suddenly cooled (resp. heated) through such boundary.

Zienkiewicz and Taylor (2000) make the mathematical model responsible of the numerical instabilities associated to thermal shocks. In fact, thermal shocks arise due to the jump between initial and boundary conditions, considered to be physically unrealistic by those authors. They propose then to apply gradually the initial conditions, avoiding in such a way the problems caused by thermal shocks on numerical modeling. Unfortunately, in many important processes such as hot forming and casting, some boundary regions suffer very high cooling or heating rates, in a period of time that is very small with respect to the discretization time step, so that the initial jump in boundary conditions really exists and must be modeled. Numerous concrete examples can be given, for instance the contact between a hot metal workpiece and forming tools at a much lower temperature, or between the flow of molten metal and the components of a casting mould.

The proper modeling of thermal shocks at a given time instant is achieved provided the layer currently affected by the thermal shock be at least one-element wide (Hogge and Gerrekens, 1982; Gerrekens, 1988; Wagoner and Chenot, 2001). This is the so-called penetration depth condition. For a mesh of uniform element size $\Delta x$, the time increment $\Delta t_{t s}$ required to satisfy this condition is given by

$$
\Delta t_{t s}=\alpha \frac{\rho c_{p}}{k} \Delta x^{2}
$$

where $\alpha$ is a constant of order 1 (Hogge and Gerrekens, 1982; Gerrekens, 1988; Wagoner and Chenot, 2001).

In a purely thermal analysis, thermal shocks have a relatively short-term effect, since the solution is no longer affected once the thermal shock layer is developed enough. However, in a coupled analysis, e.g. a thermo-mechanical analysis, the instabilities in the early stages may invalidate the whole solution, as it is the case for inelastic (history-dependent) materials with thermo-dependent mechanical properties.

We can also satisfy the penetration depth condition by refining the mesh in the concerned 
regions, as done by Sheu et al. for convection-diffusion (Sheu et al, 1999) and convectiondiffusion-reaction problems (Sheu and Chen, 2002), with satisfactory results in terms of accuracy. The adaptive refinement technique proposed by Sheu et al (1999), based on the bisection of rectangular finite elements, allows to restrict the refined grid only to the regions of large gradients, leaving the grid outside unaltered. Unfortunately, the implementation of such technique for general unstructured meshes is not as simple. Further, only 2D problems were considered in those works.

In fact, the option of mesh refinement is usually unaffordable in real 3D applications, for which the finite element grids are now frequently non-structured and composed of tetrahedral elements. Let consider for instance a metal casting problem, where an initially extremely rapid cooling process takes place at the interface between the melt and the chilled mould, requiring a highly refined mesh on both domains.

Regarding this constraint, and assuming finite element meshes not especially refined at their boundaries, let us outline then the different approaches developed to deal with thermal shocks using tetrahedral elements.

The first one arises naturally from the penetration depth condition: if the mesh must keep unchanged, a high enough time step, that is $\Delta t_{t s}$ as determined by equation (1), is needed in the early stage of the simulation. Then, by using an implicit time-stepping scheme, the heat equation can be solved taking $\Delta t_{t s}$ as time step without stability concerns. Unfortunately, $\Delta t_{t s}$ is frequently too large for an accurate integration of the heat equation and all the other conservation equations that may be coupled with it, e.g., the momentum and chemical species balances. Jaouen (1998) has proposed to adopt $\Delta t_{t s}$ as time step, and then to linearly interpolate the computed thermal solution to an adequate time step $\Delta t<\Delta t_{t s}$. This strategy, called asynchronous thermal analysis, gives satisfactory results for linear or slightly nonlinear problems, which is not the case in solidification processes.

The second approach is based on the M-matrix theory (Ortega and Rheinboldt, 1970): the satisfaction of the maximum principle requires the system matrix obtained after discretization be an M-matrix (a real, non-singular $n \times n$-matrix $\mathbf{A}$ is an M-matrix if $\mathbf{A}^{-1} \geq 0$ and all its outdiagonal components are non-positive). Putti and Cordes(1998) and Cordes and Putti (2001) proposed an Orthogonal Subdomain Collocation (OSC) technique that produces a diffusion matrix in M-matrix-form when applied to tetrahedral finite elements in a 3D Delaunay triangulation satisfying suitable conditions on the elements geometry adjacent to the boundary affected by thermal shocks. If the capacitance matrix is lumped, it becomes an M-matrix. In such a way, the system matrix for a transient diffusion problem, being the sum of two symmetric M-matrices, is also an M-matrix. It is not longer the case when the system matrix contains an advection (non-symmetric) term, and therefore this approach can not be generalized to advection-diffusion problems. In addition, the geometrical constraints on the mesh prevent from using general meshing codes, that generally use tetrahedral elements. This leads several authors (Letniowski, 1992; Kosik et al, 2000) to directly discourage the use of linear tetrahedral finite elements; Kosik et al (2000) promote to use the finite volume method (FVM) instead. However, we can not ignore the wide diffusion of FEM in the existing codes, as well as its versatility compared to FVM. 
Not only FVM but also some FEM models are free of oscillations under thermal shocks. This is the case of discontinuous Galerkin (DG) models. Pichelin and Coupez (1999) and Batkam (2002) have developed explicit and implicit DG models, respectively, to solve thermal problems on general 3D triangulations. They used P0 elements, i.e., tetrahedra with constant temperature inside. Therefore, the use of these techniques implies no more nodal but elemental unknowns. Let us remark that in a typical 3D triangulation, the number of elements is about 5-times greater than the number of vertex nodes. In other words, DG-P0 elements are not only less convenient than P1 elements in terms of the order of the discretization error, but also regarding the computational cost. In addition, the coupling with the resolution of complementary conservation equations, such as momentum or chemical species, for which a nodal solution may be obtained, yields to difficulties regarding the consistency of the different fields of variables.

The present work aims to retain the advantages of using P1 finite elements, making possible at the same time to model thermal shocks when small enough time increments are used. The diffusion-split method presented here has common items with that of Jaouen (1998) regarding the idea of satisfying the penetration depth condition, as well as with those inspired by the M-matrix theory (Putti and Cordes, 1998; Cordes and Putti, 2001) that focus on the form of the system matrix. The resulting formulation, representing just a slight modification to the standard Galerkin one, can be easily implemented into existing FEM codes.

\section{THE HEAT EQUATION}

The well-known local form of the heat equation is

$$
\rho c_{p} \frac{d T}{d t}-\nabla \cdot(k \nabla T)=Q \quad \text { in } \Omega
$$

where $\Omega$ is the analyzed domain, $t$ the time variable, $T$ the temperature, $\rho c_{p}$ the specific heat, $k$ the thermal conductivity, and $Q$ an internal heat source. First, we consider here the energy equation without possible phase change. This aspect will be discussed in section 4.2. Equation (2) is subject to the initial condition:

$$
T=T^{0} \quad \text { at } t=0
$$

and the following boundary conditions :

$$
\begin{gathered}
T=T_{w} \quad \text { on } \Gamma_{T} \\
-(k \nabla T) \cdot \mathbf{n}=q_{w} \quad \text { on } \Gamma_{q} \\
-(k \nabla T) \cdot \mathbf{n}=h\left(T-T_{e x t}\right) \quad \text { on } \Gamma_{c}
\end{gathered}
$$

prescribing the temperature $T_{w}$ on $\Gamma_{T}$, the heat flux $q_{w}$ through $\Gamma_{q}$, and the heat exchange through $\Gamma_{c}$ due to convection to the environment at temperature $T_{\text {ext }}$ with $h$ as the convection 
coefficient; $\Gamma_{T}, \Gamma_{q}$, and $\Gamma_{c}$ are non-overlapping portions of the boundary $\Gamma$ of $\Omega$, being $\mathbf{n}$ the unit normal vector pointing outwards to $\Gamma$.

\section{STANDARD FINITE ELEMENT FORMULATION}

The Galerkin FEM applied to the initial and boundary value problem defined by equations (2) to (6) yields the system of first-order differential equations (see the classical FEM literature, e.g. Zienkiewicz and Taylor, 2000, for details)

$$
\mathbf{C} \frac{d \mathbf{T}}{d t}+\mathbf{K T}-\mathbf{F}=\mathbf{0}
$$

where $\mathbf{T}$ is the vector of nodal unknown temperatures, $\mathbf{C}$ is the capacitance matrix, $\mathbf{K}$ the conductivity matrix, and $\mathbf{F}$ is the internal source and external flux vector, defined as

$$
\begin{gathered}
C_{i j}=\int_{\Omega} \rho c_{p} N_{i} N_{j} d V \\
K_{i j}=\int_{\Omega} k \nabla N_{i} \cdot \nabla N_{j} d V+\int_{\Gamma_{c}} h N_{i} N_{j} d S \\
F_{i}=\int_{\Omega} Q N_{i} d V+\int_{\Gamma_{c}} q_{w} N_{i} d S+\int_{\Gamma_{c}} h T_{e x t} N_{i} d S
\end{gathered}
$$

being $N_{i}$ the interpolation function associated with node $i$. The fully-implicit Euler-backward scheme is used to integrate equation (7) in time. Then, once the temperature at time $t$, say $T$, is known, the temperature $T$ at time $t+\Delta t$ can be obtained by solving the discrete equation

$$
\mathbf{C} \frac{\mathbf{T}-\mathbf{T}^{t}}{\Delta t}+\mathbf{K T}-\mathbf{F}=\mathbf{0}
$$

\section{THE DIFFUSION-SPLIT METHOD}

As mentioned earlier, it is known that the spurious solutions observed in case of thermal shocks are associated with the form of the system matrix (Putti and Cordes, 1998; Cordes and Putti, 2001; Kozic et al, 2000). Then, let us rewrite the governing discrete equation (11) by splitting the diffusion term as follows

$$
\mathbf{C} \frac{\mathbf{T}-\mathbf{T}^{t}}{\Delta t}+\mathbf{K}^{*} \mathbf{T}-\mathbf{F}=\mathbf{S}
$$

where

$$
\mathbf{S}=\left(\mathbf{K}^{*}-\mathbf{K}\right) \mathbf{T}
$$

with 


$$
K_{i j}^{*}=\int_{\Omega} k^{*} \nabla N_{i} \cdot \nabla N_{j} d V+\int_{\Gamma_{c}} h N_{i} N_{j} d S
$$

Now, assuming $\mathbf{S}$ to be an explicit source term, an augmented conductivity $k^{*}$ can be defined to satisfy the penetration depth condition for $\Delta t$ as the first time step:

$$
k^{*}= \begin{cases}k & \text { if } \Delta t_{t s} \leq \Delta t \\ k \frac{\Delta t_{t s}}{\Delta t} & \text { if } \Delta t_{t s}>\Delta t\end{cases}
$$

The value of $k^{*}$ decreases with time from the value given by equation (15) at $t=0$ to the real conductivity $k$ when $t+\Delta t \geq \Delta t_{t s}$. Therefore, in the latter case, equations (11) and (12) are identical. Regarding the source term $\mathbf{S}$, an explicit approximation is built by taking a known value of $\mathbf{T}$ in equation (13), $\mathbf{T}^{t}$ being the best choice since any approximation to $\mathbf{T}$ obtained using an explicit time-stepping scheme may be affected by instabilities. Thus,

$$
\mathbf{S} \approx\left(\mathbf{K}^{*}-\mathbf{K}\right) \mathbf{T}^{t}
$$

It is interesting to note that during the early stages of the simulation, there is no sensible variation of the temperature outside those regions under thermal shocks, and hence the approximation implied by equation (16) is local and temporary.

\subsection{The penetration depth condition on unstructured meshes}

For non-uniform unstructured meshes, the computation of $\Delta t_{t s}$ using equation (1) complicates due to the uncertain definition of the mesh size $\Delta x$. Jaouen (1998) explores all the non-adiabatic boundaries of the analyzed domain, determining the minimum, the maximum, and the average value of $\Delta x$, the choice of the adequate value left to the user's expertise. Being the square value of $\Delta x$ involved in equation (1), the influence of this choice on the proper modeling of thermal shocks is crucial. According to our practical experience, $\Delta t_{t s}$ is underestimated when computed with the minimum $\Delta x$, and hence the penetration depth condition is clearly not satisfied in some regions. On the other hand, the use of the maximum $\Delta x$, leading to an overestimation of $\Delta t_{t s}$, has a detrimental effect on the accuracy of the diffusion split method, producing an excessively large $k^{*}$. Using the average mesh size, the result is uncertain.

If it were possible to identify a priori those regions under thermal shocks, and the mesh within each region were quite uniform, then we could define a local $\Delta t_{t s}$ (and hence a local $k^{*}$ ). Anyway, for the sake of simplicity, we prefer to determine a global $\Delta t_{t s}$. It is worth noting that in the case of monotonic cooling, $\Delta t_{t s}$ can be easily determined by solving equation (12) for increasing $\Delta t_{t s}$ until the computed temperature not exceed the previous one at each node of the mesh. This should not take more than a few iterations to obtain an accurate enough value of $\Delta t_{t s}$. Normally, this is made only once at the beginning of the simulation, so the additional computational cost is negligible in practice. 


\subsection{The diffusion-split method for solidification problems}

In the case of liquid-solid phase change, we introduce the enthalpy function

$$
H(T)=\int_{0}^{T} \rho c_{p}(T) d T+g_{l} \rho L
$$

where $g_{l}$ is the volumetric fraction of liquid $\left(0 \leq g_{l} \leq 1, g_{l}=0\right.$ in the solid, $g_{l}=1$ in the liquid), assumed to be a given function of the temperature, and $L$ the specific latent heat of solidification. From the enthalpy function, we can derive an effective heat capacity $\rho c_{\text {eff }}$, which accounts for phase change effects. Assuming that $g_{l}$ is a function of the temperature only, for the sake of simplicity, it is defined as:

$$
\rho c_{e f f}=\frac{\partial H}{\partial T}=\rho c_{p}+\rho L \frac{d g_{l}}{d T}
$$

subject to the initial condition (3) and the boundary conditions (4) to (6). In general, Equation (18) is only valid in a weak sense, since the first term resembles the Dirac's delta function for eutectic or isothermal transformations. The direct evaluation of $\rho c_{\text {eff }}$ as done in the early solidification models (Bonacina et al, 1973) yields highly inaccurate results in problems with a narrow solidification range unless in a huge number of sampling points be placed in each element affected by phase change. For this reason, $\rho c_{\text {eff }}$ is usually regularized as proposed for instance by Lemmon $(1979,1981)$ :

$$
\rho c_{e f f}=\frac{\|\nabla H\|}{\|\nabla T\|}
$$

where $\|(*)\|$ is the norm of vector $(*)$, and spatial regularization is obtained by assuming $H$ interpolated in the same way as $T$. Formula (18) yields an element-wise constant effective heat capacity for linear tetrahedral and triangular elements. Now the discrete heat equation involving phase change can be written as:

$$
\mathbf{C}_{e f f} \frac{\mathbf{T}-\mathbf{T}^{t}}{\Delta t}+\mathbf{K T}-\mathbf{F}=\mathbf{0}
$$

whose form is identical to that of the original equation, only requiring to replace $\rho c_{p}$ by $\rho c_{e f f}$ in the definition of the heat capacitance matrix $\mathbf{C}$, equation (7).

In consequence, the implementation of the diffusion-split method in this case is essentially the same as described above, except for determining $\Delta t_{t s}$, which can not in general be estimated a priori. In the elements undergoing phase change, $\rho c_{\text {eff }}$ is considerably greater than $\rho c_{p}$. Also, it is highly variable with time. In this case, $\Delta t_{t s}$ should be determined at each time step until thermal shocks effects completely disappear. Actually, a general and efficient procedure to determine $\Delta t_{t s}$ for non-linear problems and unstructured meshes remains a 
research item.

\section{APPLICATIONS}

\subsection{Test case: one-dimensional cooling}

Let us consider first the one-dimensional case of a semi-infinite domain, initially at the uniform temperature $T^{0}=800^{\circ} \mathrm{C}$, whose surface temperature suddenly falls to a value $T_{w}=25^{\circ} \mathrm{C}$, kept constant. Table I lists the material properties. This problem illustrates some typical features of thermal analysis in steel hot forming. We use a 3D structured triangulation with uniform element size $\Delta x=2 \mathrm{~mm}$ in the flux direction. A constant time step $\Delta t=0.1 \mathrm{~s}$ is adopted.

\section{Take in Table I}

In this case, the original Galerkin solution is affected by thermal shocks, as evidenced by the spurious temperature increment of $13.2^{\circ} \mathrm{C}$ at the first time step for a node located $4 \mathrm{~mm}$ far from the cooled wall (Figure 1).

\section{Take in Figure 1}

For linear tetrahedral finite elements and consistent (not-lumped) capacitance matrix, the constant $\alpha$ in equation (1) is taken equal to unity (Jaouen, 1998), obtaining then $\Delta t_{t s}=0.748 \mathrm{~s}$. However, using the procedure described in the preceding section, every nodal point is already free of unphysical heating for $\Delta t_{t s}=0.544 \mathrm{~s}$. And, by tolerating a small temperature overshoot of $0.1^{\circ} \mathrm{C}$ for instance, this value reduces to $0.287 \mathrm{~s}$. The augmented conductivity $k^{*}$ is computed using equation (15) for the first time step, and decreases linearly with time until reach the original value $k$ for $t \geq \Delta t_{t s}$. Let us remind that the closer the values of $\Delta t$ and $\Delta t_{t s}$, the lesser the artificial increment of the conductivity in the thermal regions when the diffusion split method is used. This is clearly evident in Figure 1 where the temperature rate at the first time step increases as $k^{*}$ does, which is an unphysical but numerical effect of the diffusion split method.

\subsection{Ingot solidification}

We consider now the ingot casting model shown in Figure 2. The material properties of each component and other model parameters are listed in Table II. For the sake of simplicity, the solid fraction is supposed be linear between the liquidus and solidus temperature. The model is axisymmetric, and a small sector of $12^{\circ}$ is considered for $3 \mathrm{D}$ simulation. The ingot has a radius of $0.433 \mathrm{~m}$ at the top, and its total height is $2.58 \mathrm{~m}$. This is a typical simulation illustrating the problems posed by thermal shocks in current $3 \mathrm{D}$ applications.

Take in Figure 2 
First, due to the very different diffusion properties of the different components, an excessively large time step is needed in order to satisfy the penetration depth condition. Taking into account the average element size (since the mesh density is quite uniform in each subdomain), equation (1) yields the values of $\Delta t_{t s}$ listed in Table II.

\section{Take in Table II}

For $\Delta t=0.1 \mathrm{~s}$ (an adequate value of the time increment for the early stages of the simulation), the obtained solution using standard Galerkin FEM with P1 elements is completely useless, exhibiting nodal temperatures that are $94^{\circ} \mathrm{C}$ above the initial temperature in the ingot. The solution is even worse in the domain of smallest diffusivity: in the casting powder, this spurious overheating attains $320^{\circ} \mathrm{C}$, while negative temperatures are observed in the insulator.

In order to apply the diffusion-split solution, we choose to admit an overheating of $0.5^{\circ} \mathrm{C}$ in the ingot at the first time step. In such a way, the value of $\Delta t_{t s}$ falls to $11 \mathrm{~s}$, and considerably less artificial diffusion is added. A reference solution is obtained using the above mentioned explicit Taylor discontinuous Galerkin (TDG) method with P0 triangular elements (Pichelin and Coupez, 1999), probed to be free of instabilities caused by thermal shocks.

Figure 3 shows the temperature through a cross section of the ingot, situated $1.60 \mathrm{~m}$ far from the bottom, at the beginning of simulation $(t=1.1 \mathrm{~s})$. A good agreement between diffusion-split and TDG solutions is observed. The points in the plot correspond to the center of the TDG elements, while they are nodal values for the 3D triangulation, letting us note that the two-dimensional TDG mesh is about 2.5-times denser than the 3D mesh in this region.

\section{Take in Figure 3}

Once the solidification has progressed in the ingot, the agreement between both models remains satisfactory, as evidenced in Figure 4 for the temperature field at time $t=20 \mathrm{~min}$ and $t=2 \mathrm{~h}$. The slight differences can be attributed to the effects of nodal smoothing, which is a necessary post-treatment in the case of TDG calculations, and to the differences in mesh densities.

Take in Figure 4

\section{CONCLUSIONS}

The diffusion-split method makes possible to solve problems involving thermal shocks using the FEM with linear tetrahedral elements. Compared to the previous models using P1 elements, the diffusion split method works for general meshes, contrary to the OSC-FEM method (Putti and Cordes, 1998; Cordes and Putti, 2001), and the heat equation is solved for an adequate time step (i.e. not excessively large), contrary to the so-called asynchronous 
analysis (Jaouen, 1998). Compared to the discontinuous Galerkin methods using P0 elements (Pichelin and Coupez, 1999; Batkam, 2002), the present model has not only a better accuracy order, but also a smaller computational cost. The present model can be implemented in most existing FEM codes with a minimal effort.

\section{ACKNOWLEDGEMENTS}

The support of Arcelor and Ascometal companies, and of the french Ministère de l'Economie, des Finances et de l'Industrie in the frame of the OSC-Continuous Casting project, is acknowledged.

\section{REFERENCES}

Batkam, S. (2002), Thermique multidomaine en simulation numérique du remplissage 3D. $\mathrm{PhD}$ Thesis, École des Mines de Paris, France.

Bonacina, C., Comini, G., Fasano, A. and Primicerio, M. (1973), "Numerical solution of phase-change problems", International Journal of Heat and Mass Transfer, Vol. 16, pp. 18251832 .

Cordes, C. and Putti, M. (2001), "Accuracy of Galerkin finite elements for groundwater flow simulations in two and three-dimensional triangulations", International Journal for Numerical Methods in Engineering, Vol. 52, pp. 371-387.

Gerrekens, P. (1988), Modélisation par éléments finis des phénomènes d'ablation thermique avec pyrolyse, PhD Thesis, Faculté des Sciences Appliquées, Université de Liège, Belgium.

Hogge, M. and Gerrekens, P. (1982), "Steep gradient modeling in diffusion problems", in Lewis, R. W. et al (Eds.), Numerical Methods in Heat Transfer, Vol. II, John Wiley Publications, Chichester, pp. 73-97.

Jaouen, O. (1998), Modélisation tridimensionnelle par éléments finis pour l'analyse thermomécanique du refroidissement des pièces coulées, PhD Thesis, École des Mines de Paris, France.

Kosik, R., Fleischmann, P., Haindl, B., Pietra, P. and Selberherr, S. (2000), "On the interplay between meshing and discretization in three-dimensional diffusion simulation", IEEE Transactions on Computer-Aided Design of Integrated Circuits and Systems, Vol. 19 No. 11, pp. 1233-1240.

Lemmon, E.C. (1979), "Phase-change techniques for finite element conduction codes", in Lewis, R. W. and Morgan, K. (Eds.), Numerical Methods in Thermal Problems, pp. 149-158, Pineridge Press, Swansea, UK.

Lemmon, E.C. (1981), Multidimensional integral phase change approximations for finite 
element conduction codes, in Lewis, R. W., Zienkiewicz, O. C. and Morgan, K. (Eds.), Numerical Methods in Heat Transfer, Chapter 10, pp. 201-213, John Wiley \& Sons, New York

Letniowski, F. W. (1992), “Three-dimensional Delaunay triangulations for finite element approximations to a second-order diffusion operator", SIAM Journal on Scientific and Statistical Computing, Vol. 13 No. 3, pp. 765-770.

Ortega, J. M. and Rheinboldt, W. C. (1970), Iterative solution of nonlinear equations in several variables, Academic Press, New York.

Pichelin, E. and Coupez T. (1999), "A Taylor discontinuous Galerkin method for the thermal solution in 3D mold filling", Computer Methods in Applied Mechanics and Engineering, Vol. 178, pp. 153-169.

Putti, M. and Cordes, C. (1998), "Finite element approximation of the diffusion operator in tetrahedral”, SIAM Journal on Scientific Computing, Vol. 19 No. 4, pp. 1154-1168.

Sheu, T. W. H., Fang, C. C., Tsai, S. F. and Huang, C.-Y. (1999), "On an adaptive monotonic convection-diffusion flux discretization scheme", Computer Methods in Applied Mechanics and Engineering, Vol. 173, pp. 201-215.

Sheu, T. W. H. and Chen, H. Y. H. (2002), "A multi-dimensional monotonic finite element model for solving the convection-diffusion-reaction equation", International Journal for Numerical Methods in Fluids, Vol. 39, pp. 639-656.

Wagoner, R. H. and Chenot, J.-L. (2001), Metal forming analysis, Cambridge University Press, Cambridge, UK.

Zienkiewicz, O. C. and Taylor, R. L. (2000), The finite element method - The basis, Butterworth-Heinemann, Oxford. 


\section{TABLES}

Table I: Material properties for the one-dimensional cooling problem.

\begin{tabular}{|l|c|}
\hline Density $\rho$ & $7800 \mathrm{~kg} / \mathrm{m}^{3}$ \\
\hline Heat capacity $c_{p}$ & $360 \mathrm{~J} /\left(\mathrm{kg}{ }^{\circ} \mathrm{C}\right)$ \\
\hline Thermal conductivity $k$ & $15 \mathrm{~W} /\left(\mathrm{m}^{\circ} \mathrm{C}\right)$ \\
\hline
\end{tabular}

Table II: Material properties and simulation data for the ingot solidification problem.

\begin{tabular}{|c|c|}
\hline \multicolumn{2}{|l|}{ Ingot } \\
\hline Density $\rho$ & $7450 \mathrm{~kg} / \mathrm{m}^{3}$ \\
\hline Heat capacity $c_{p}$ & $510 \mathrm{~J} /\left(\mathrm{kg}^{\circ} \mathrm{C}\right)$ \\
\hline Thermal conductivity $k$ & $30 \mathrm{~W} /\left(\mathrm{m}^{\circ} \mathrm{C}\right)$ \\
\hline Latent heat $L$ & $241000 \mathrm{~J} / \mathrm{kg}$ \\
\hline Solidus temperature & $1432^{\circ} \mathrm{C}$ \\
\hline Liquidus temperature & $1454^{\circ} \mathrm{C}$ \\
\hline Initial temperature & $1534^{\circ} \mathrm{C}$ \\
\hline Time step $\Delta t_{t s}$ & $20.3 \mathrm{~s}$ \\
\hline \multicolumn{2}{|l|}{ Mold } \\
\hline Density $\rho$ & $7200 \mathrm{~kg} / \mathrm{m}^{3}$ \\
\hline Heat capacity $c_{p}$ & $600 \mathrm{~J} /\left(\mathrm{kg}^{\circ} \mathrm{C}\right)$ \\
\hline Thermal conductivity $k$ & $30 \mathrm{~W} /\left(\mathrm{m}^{\circ} \mathrm{C}\right)$ \\
\hline Initial temperature & $80^{\circ} \mathrm{C}$ \\
\hline Time step $\Delta t_{t s}$ & $36.3 \mathrm{~s}$ \\
\hline \multicolumn{2}{|l|}{ Insulator } \\
\hline Density $\rho$ & $780 \mathrm{~kg} / \mathrm{m}^{3}$ \\
\hline Heat capacity $c_{p}$ & $848.5 \mathrm{~J} /\left(\mathrm{kg}^{\circ} \mathrm{C}\right)$ \\
\hline Thermal conductivity $k$ & $0.82 \mathrm{~W} /\left(\mathrm{m}^{\circ} \mathrm{C}\right)$ \\
\hline Initial temperature & $60^{\circ} \mathrm{C}$ \\
\hline Time step $\Delta t_{t s}$ & $41.3 \mathrm{~s}$ \\
\hline \multicolumn{2}{|l|}{ Casting powder } \\
\hline Density $\rho$ & $500 \mathrm{~kg} / \mathrm{m}^{3}$ \\
\hline Heat capacity $c_{p}$ & $1100 \mathrm{~J} /\left(\mathrm{kg}^{\circ} \mathrm{C}\right)$ \\
\hline Thermal conductivity $k$ & $0.35 \mathrm{~W} /\left(\mathrm{m}^{\circ} \mathrm{C}\right)$ \\
\hline Initial temperature & $1534^{\circ} \mathrm{C}$ \\
\hline Time step $\Delta t_{t s}$ & $70.3 \mathrm{~s}$ \\
\hline \multicolumn{2}{|l|}{ Interfaces between materials } \\
\hline Heat exchange coefficient $h$ & $1000 \mathrm{~W} /\left(\mathrm{m}^{20} \mathrm{C}\right)$ \\
\hline \multicolumn{2}{|l|}{ Interfaces with air } \\
\hline Heat exchange coefficient $h$ & $30 \mathrm{~W} /\left(\mathrm{m}^{20} \mathrm{C}\right)$ \\
\hline External temperature $T_{e x t}$ & $20^{\circ} \mathrm{C}$ \\
\hline
\end{tabular}




\section{FIGURES}

Figure 1: One-dimensional academic test for thermal shock. Early evolution of the temperature at a node situated $4 \mathrm{~mm}$-far from the chilled wall.

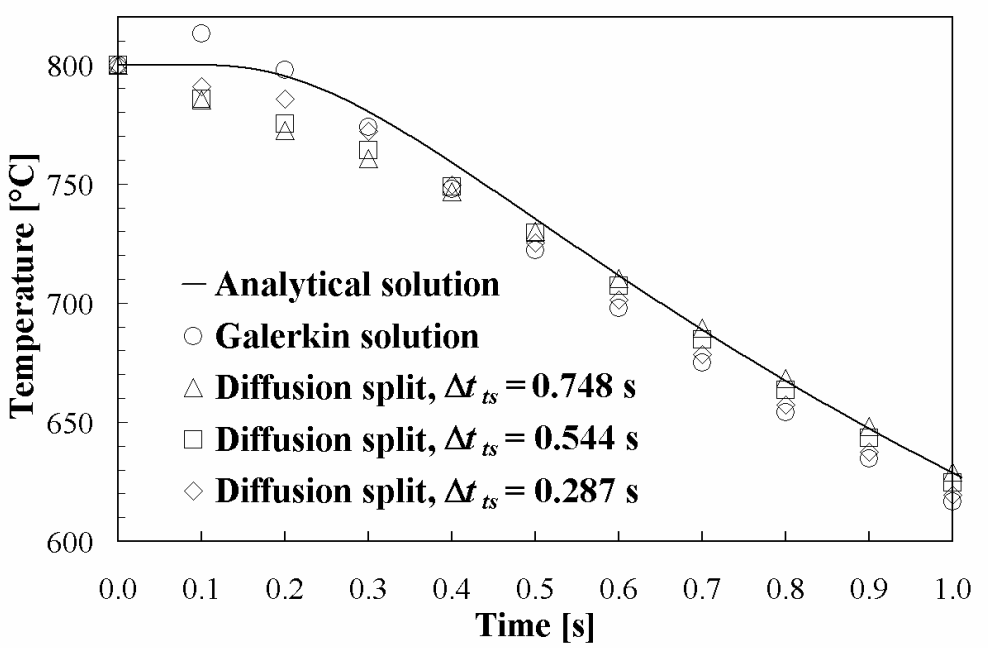


Figure 2: Industrial application test. FEM model of an ingot casting process.
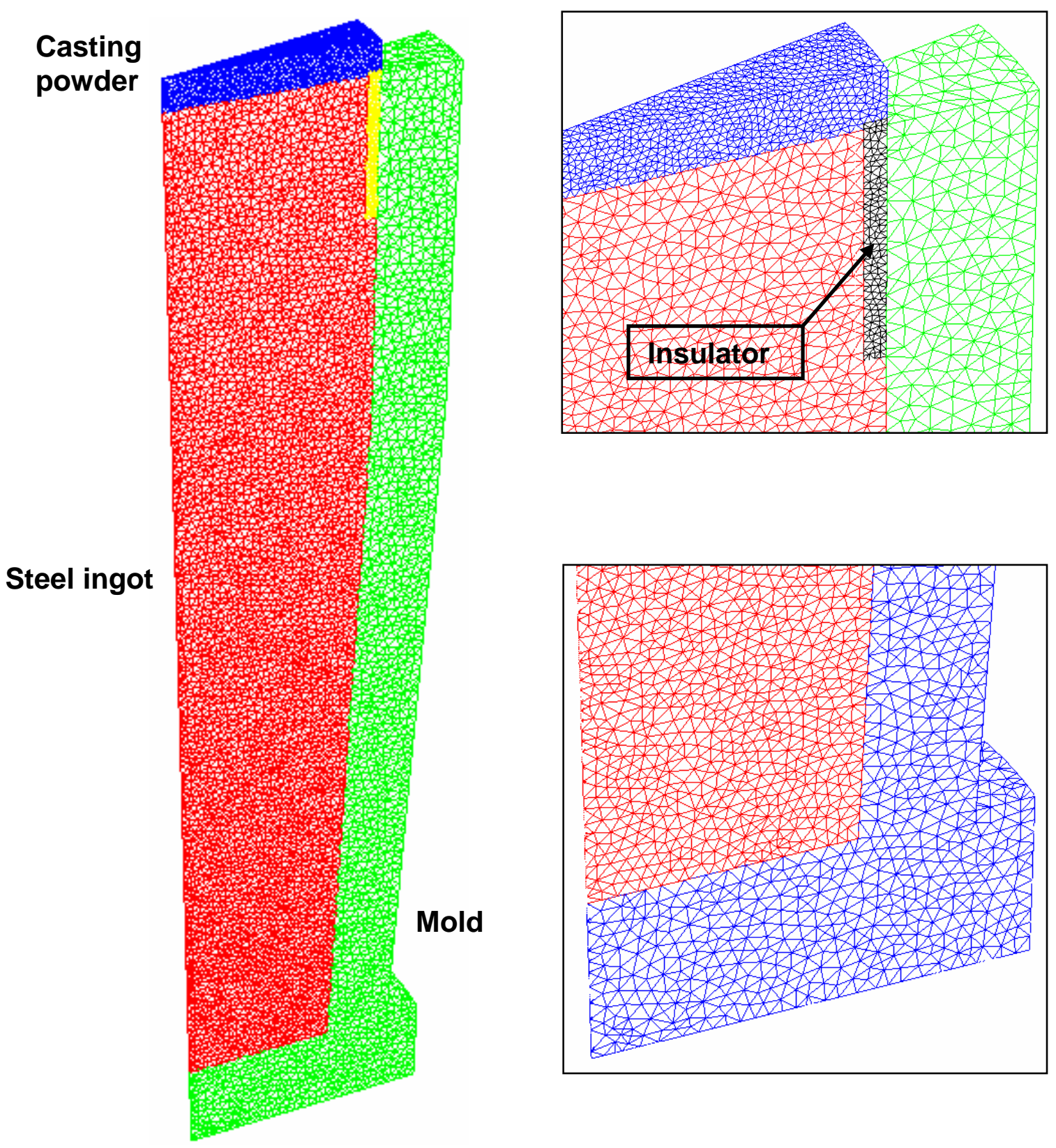
Figure 3: Temperature in the ingot at time $1.1 \mathrm{~s}$ in a cross section located $1.6 \mathrm{~m}$-far from the ingot bottom.

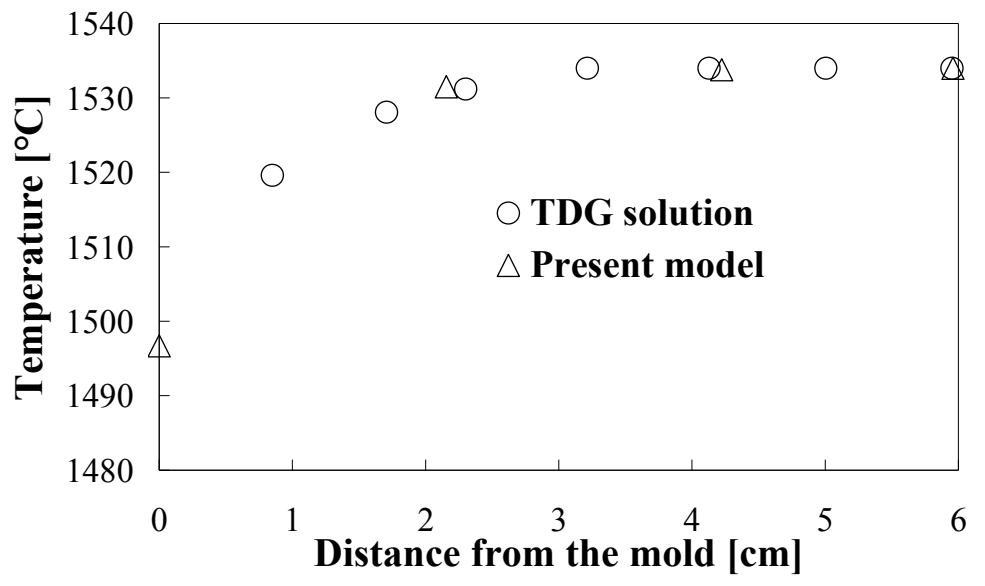


Figure 4: Temperature in the ingot after $20 \mathrm{~min}$ (left) and 2 hours (right). On the left side ingot sections, the temperature distribution obtained with the proposed diffusion-split method (3D calculation). On the right side, the same distribution as obtained by the explicit TDG method (2D calculation).
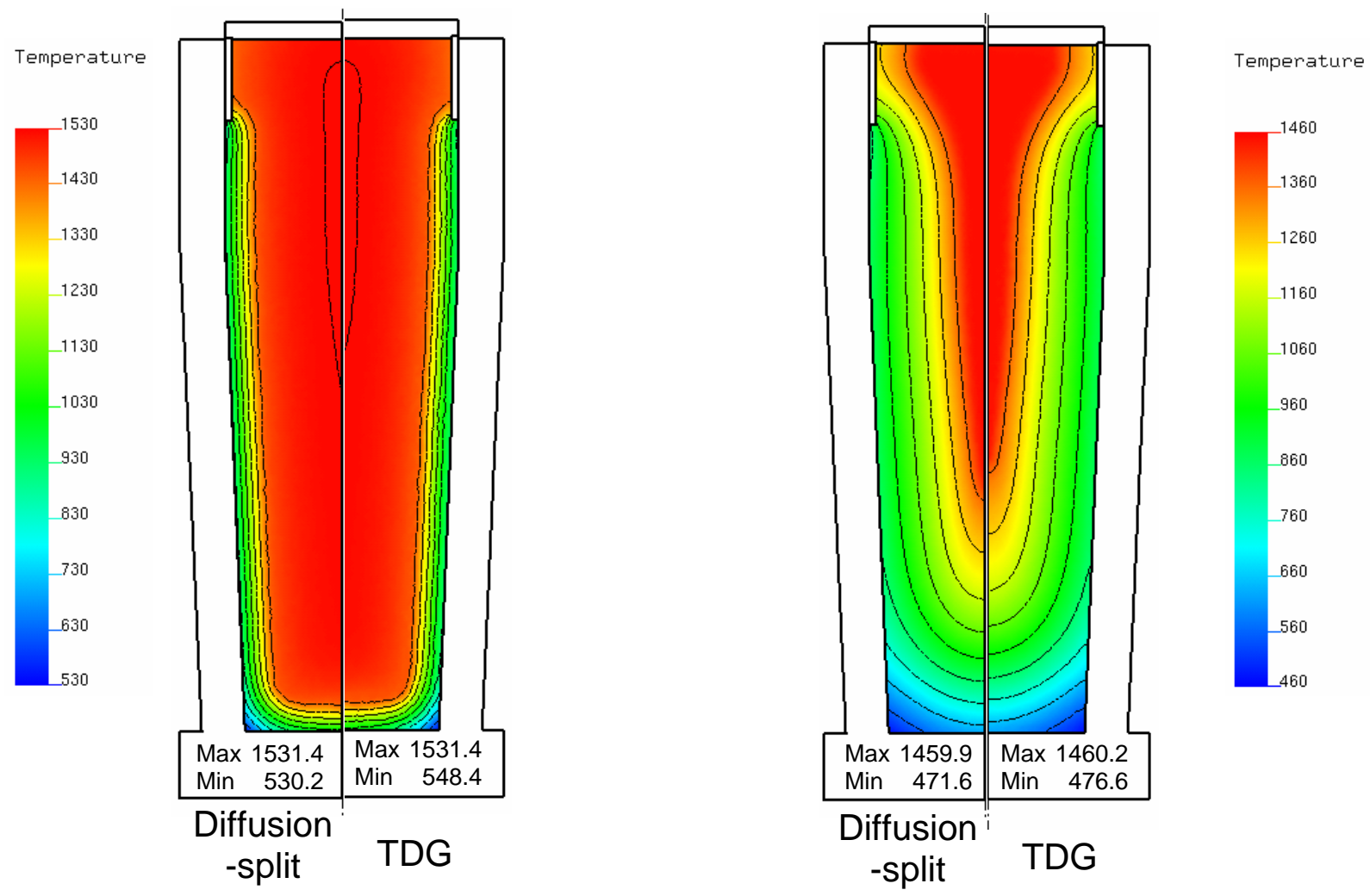


\section{BIOGRAPHIES}

Dr. Victor Daniel Fachinotti is Assistant Researcher from the Argentine Council for Scientific and Technical Research (CONICET) at the International Center for Computational Methods in Engineering (CIMEC, Argentine). He is also ad-joint professor from the National Technological University (UTN, Argentine). He obtained the "Doctor in Engineering"-degree from the National Littoral University (UNL, Argentine) in 2001 with a thesis on the thermomechanical modeling of continuous casting processes. From 2002 to 2004, he has done a postdoctoral stage at the Material Processing Center (CEMEF), Ecole des Mines de Paris, France, under the direction of Dr. M. Bellet. His main interest is the modeling of solidification processes, mainly casting processes, including solid mechanics, fluid dynamics, heat transfer and macrosegregation.

E-mail: vfachino@intec.unl.edu.ar

Address:

Centro Internacional de Métodos Computacionales en Ingeniería (CIMEC)

Parque Tecnológico del Litoral Centro

Ruta Nacional 168 - Paraje El Pozo (S3007ABA) Santa Fe, Argentine

Dr. Michel Bellet is the head of the research group "Thermomechanics and Plasticity" at the Materials Processing Center (CEMEF) of Ecole des Mines de Paris. His research interests deal with the numerical modelling of coupled thermomechanical problems occurring in industrial forming processes for metals and polymers, with a special focus on metal casting processes..

E-mail: michel.bellet@ensmp.fr

Address:

Centre de Mise en Forme des Matériaux (CEMEF)

BP 207

F-06904 Sophia Antipolis, France 